\title{
Pour une autre science sociale
}

\section{Gérald Berthoud}

\section{OpenEdition \\ Journals}

Édition électronique

URL : http://journals.openedition.org/ress/501

DOI : 10.4000/ress.501

ISSN : 1663-4446

\section{Éditeur}

Librairie Droz

\section{Édition imprimée}

Date de publication : 1 décembre 2003

ISBN : 2-600-00912-4

ISSN : 0048-8046

\section{Référence électronique}

Gérald Berthoud, «Pour une autre science sociale», Revue européenne des sciences sociales [En ligne], XLI-127 | 2003, mis en ligne le 27 novembre 2009, consulté le 20 avril 2019. URL : http:// journals.openedition.org/ress/501; DOI : 10.4000/ress.501 


\section{Gérald BERTHOUD}

\section{POUR UNE AUTRE SCIENCE SOCIALE}

Tel était le thème du $\mathrm{XX}^{\mathrm{e}}$ colloque annuel organisé par le Groupe d'Etude «Pratiques sociales et Théories » ${ }^{1}$. Pour orienter les communications et les discussions, un texte de présentation avait été distribué à l'avance. Il est repris ici in extenso:

«La fin des certitudes, le déclin des vérités, la crise de la raison, l'échec des grandes idéologies ont transformé nos vies en spectacles et même en simulacres. Les sciences humaines et sociales sont désemparées face à une socialité éclatée. Sans valeurs partagées, nos existences individuelles et collectives sont privées de sens.

Faut-il s'accommoder d'une telle situation, s'enfermer dans le confort de l'érudition académique ou céder aux tentations scientistes? Peut-on alors prétendre à une connaissance intégrale des sociétés, quand nos savoirs sont partiaux et ethnocentriques, méfiants à l'égard des valeurs antagonistes, des représentations diverses du social, des sentiments et des désirs qui travaillent des formes de socialité refoulées? Faut-il continuer à faire confiance aux sciences sociales dont le souci majeur semble être celui de redoubler la vision économique du monde, de naturaliser la réalité culturelle qu'elles devraient au contraire dévoiler? Comment comprendre et expliquer l'émergence du nouveau, saisir ce qui est autre alors que le causalisme ramène le complexe à l'identique et au banal, alors que l'objectivisme réduit le discontinu au continu et les multiples dimensions de l'expérience sociale aux seuls aspects mesurables ou formalisables? Le colloque voudrait ainsi aborder une question majeure. Celle de savoir si la démarche comparative, fondée sur des observations interculturelles et transhistoriques et la pratique du doute méthodologique ne constituent pas les pré-conditions d'une science sociale autre?

Le contraste entre nous et les autres, aujourd'hui et autrefois, montre certes l'originalité de notre société, mais aussi des permanences propres à l'ensemble de

Cette rencontre s'est déroulée à l'Institut d'anthropologie et de sociologie de l'Université de Lausanne, les 12 et 13 juin 2003. Huit intervenants ont présenté, au cours de la première journée, des exposés qui sont repris ici. La deuxième journée a pris la forme d'un «colloque fermé». Aussi bien les contributions individuelles que l'ensemble des débats et des points de vue auraient dû être repris, discutés et approfondis, dans cette introduction. Et surtout le thème général du colloque aurait dû être clarifié et explicité. Malheureusement ce travail de synthèse, guère possible dans un temps relativement court, n'a pas pu être mené à terme. Sous sa forme actuelle, il n'est qu'une rapide ébauche pour respecter les exigences impératives d'un programme de publications. 
l'humanité. Nous pourrions ainsi comprendre comment le mode de représentation du réel contribue en même temps à construire la réalité sociale et pourquoi les normes de comportement excluent que d'autres voies soient possibles et d'autres alternatives envisageables. En d'autres termes, l'imaginaire des sociétés échapperait-il aux divers déterminismes?

Le colloque pourrait encore s'engager dans une analyse critique des savoirs dominants, dans leur constitution, leurs résultats et leurs fonctions sociales. D'autre part, en recherchant une autre manière de voir le réel dans ses multiples dimensions, il aurait également l'ambition d'établir un inventaire critique des pratiques sociales (actions finalisées ou mode de communication totalisant; champ exclusif de la rationalité ou pluralité de significations ; phénomènes formalisés ou expressions d'une complexité irréductible).

Toutefois cette insistance sur les pratiques sociales, dans toutes leurs singularités, dans la richesse de leurs expressions comme dans leurs banalités quotidiennes, ne signifie pas le choix exclusif d'une orientation impressionniste, phénoménologique ou encore subjective. Au contraire, la réflexion sur le réel doit s'inscrire dans un mouvement dialectique propre à rendre compte des structures et des pratiques sociales dans leur réalité ou leur potentialité créatrices.

C'est dire que la critique du regard simplificateur des fonctionnalismes et des déterminismes ne devrait pas nous entraîner dans une réduction inverse. Nous croyons au contraire à la possibilité d'élaborer une science sociale qui accepte pleinement la créativité de l'imaginaire et du symbolique. Pourraient ainsi coexister la raison abstraite de la tradition culturelle dominante et les théories universalisantes avec une lecture compréhensive de la réalité sociale, sensible à la pluralité des mondes et à la vie $»^{2}$.

En principe, chaque intervenant a présenté sa contribution sur l'un ou l'autre aspect de ce texte de présentation. Pour les débats, ce même texte a fourni trois interrogations ou trois thèmes relativement bien circonscrits, pour éviter le risque d'une dispersion:

- le social et l'idée de société comme objet propre des sciences sociales;

- le social à l'épreuve de la (post)modernité;

- la démarche comparative: condition d'une science sociale autre?

Inutile d'insister sur le fait qu'il aurait fallu rendre compte de la cohérence de ces trois interrogations pour évaluer sans concession nos savoirs, sans omettre la diversité des points de vue et surtout l'existence de positions méthodologiques et théoriques radicalement différentes, telles qu'elles sont clairement apparues dans les exposés et dans les discussions tout au long du colloque. Et pourtant je ne peux qu'avancer de brèves remarques, en effleurant au mieux quelques aspects de ces interrogations fondamentales.

Ce texte de présentation est une version légèrement modifiée d'un «manifeste» du Groupe d'Etude «Pratiques sociales et Théories» (voir Berthoud et Busino 1995: 22). 


\section{LE SOCIAL ET L'IDÉE DE SOCIÉTÉ COMME OBJET PROPRE DES SCIENCES SOCIALES}

Une première interrogation inévitable, mais lourde de désaccords, porte sur l'irréductible dimension sociale de la condition humaine. Ce qui devrait nécessairement conduire à discuter la notion de société, cette catégorie centrale de la tradition sociologique. Aussi avant même de se pencher sur les multiples «problèmes de la société», c'est l'idée même de société qu'il faut s'efforcer d'expliciter. Pour s'engager dans une telle voie, plusieurs exigences s'imposent immédiatement. A commencer par distinguer clairement les notions conjointes du social et de la société comme catégories du sens commun ${ }^{3}$ et comme concepts d'une vision savante. Mais surtout, il importe de poser dès le départ l'existence de plusieurs visions savantes, qui s'affichent le plus souvent comme exclusives, en niant ainsi leur possible complémentarité.

Trois visions, plus ou moins bien attestées, s'imposent. Deux d'entre elles, au niveau de leurs principes surtout, sont depuis longtemps l'objet de discussions, au point d'enfermer les débats dans un va-et-vient permanent entre elles (voir, entre autres, Cuin 2002). Tels sont, selon des expressions largement reconnues, l'individualisme méthodologique et le holisme. Mais quand il s'agit de leur mise en œuvre, dans des recherches empiriques, les credos individualiste et holiste ne présentent plus la même cohérence. Le fait même de savoir si l'individu devrait être le point de départ de l'analyse, ou au contraire la société, apparaît clairement comme une impasse. En dehors des limites étroites d'une théorisation, une telle question est dépourvue de sens. Même pour les tenants d'un individualisme méthodologique affirmé avec force, la figure de l'individu n'est guère concevable sans la moindre allusion à un contexte. De même, un holisme pur est introuvable, sauf dans les représentations proprement caricaturales de ceux qui voient dans la démarche individualiste la seule vérité méthodologique. En bref, penser le rapport social à partir d'une totalité ou d'un individu, posés, chacun à leur manière, comme une antériorité logique, paraît inconcevable.

La tendance générale à enfermer l'opposition individu et société dans une dichotomie relève donc d'une simplification trompeuse. Elle pose, entre autres, que la société serait une réalité extérieure aux individus. Mais tout être humain est de manière irréductible un être social. Inutile d'insister sur la difficulté majeure de rendre compte d'une telle tension, propre à toute personne, entre ce qui relève de sa volonté et des règles, des normes et des valeurs qu'elle a intériorisées. Tout être humain, aussi individualisé soit-il, implique un ensemble d'institutions propres à inscrire ses actions et ses idées dans un univers normatif plus ou moins bien

Le sens commun de société et des termes qui lui sont liés, comme social, sociétal, sociabilité, ou encore socialité, renvoie à l'idée générale d'association ou de réunion, marquées par des relations durables. Mais déjà au niveau du sens commun, l'idée du social est ambiguë. Comme terme englobant, elle qualifie l'ensemble des relations constitutives d'une entité collective ou d'une société. Mais comme terme plus spécialisé, elle circonscrit une dimension particulière de la vie collective, vue comme extérieure aux sphères économique et politique. Telles sont les références, entre autres, au mouvement social, à la sécurité sociale, au travail social, ou encore au service social. Une ambiguïté qui se retrouve au niveau de la vision savante du social. 
défini ${ }^{4}$. Autant dire que la question de savoir qui, de l'individu ou de la société, est premier, est dépourvue de sens, sauf à s'enfermer dans une perspective proprement dichotomique (voir, par exemple, Elias 1991: 54).

Ce qui revient à dire que toute tentative pour expliquer et comprendre l'être humain dans son individualité comme dans sa socialité se heurte à d'évidentes limites. Partir de l'individu ou de la totalité sociale pourrait s'envisager au mieux comme une vérité partielle. C'est dire combien l'individualisme méthodologique et le holisme peuvent apparaître comme deux positions extrêmes, pour saisir le monde humain dans toute sa complexité. Si l'on en vient à accepter qu'entre l'individu et la société il n'y a rigoureusement aucune antériorité chronologique et logique $^{5}$, la seule voie équilibrée est celle qui s'inscrit dans les limites d'une vision relationnelle. En d'autres termes, réfléchir sur «la part que joue la vie sociale dans la vie humaine» (Mauss 1996: 236) équivaut à privilégier l'idée de relation. Ou encore le social correspond, entre autres, à un système de relations. A l'encontre d'une démarche de type naturaliste 6 , le savoir sur la composante sociale de la condition humaine doit considérer un ensemble de relations propres à tenir les hommes ensemble. L'objet de connaissance n'est donc pas le monde comme tel, mais l'être humain dans sa relation nécessaire avec le monde, avec les autres et avec lui-même. Plus encore, aucune expérience humaine ne peut s'inscrire dans l'immédiateté. Même dans la relation de soi à soi, l'existence d'un tiers renvoie à une instance institutionnelle ${ }^{7}$. Tout tiers symbolise un ensemble de valeurs partagées. Mais comme entre-deux, ou comme médiation, le tiers unit et sépare tout à la fois ${ }^{8}$.

\section{LE SOCIAL \\ À L'ÉPREUVE DE LA (POST)MODERNITÉ}

Mais dans le monde dit (post)moderne, l'idée même de «faire société», à travers diverses formes de socialité, est soumise à de nombreuses critiques. On en vient ainsi à considérer que le concept de société, ou encore la vision relationnelle du social, ont bien occupé une place centrale dans le développement des sciences

La notion d'institution doit être envisagée dans le sens large de «règles publiques d'action et de pensée», selon la perspective clairement avancée par Mauss (1968: 25). A suivre, par exemple, Ricœur, « le langage est la grande institution - l'institution des institutions - qui nous a chacun dès toujours précédé» (1985: 400).

5 Voir Piaget pour qui ce problème de l'individu et de la société revient à se demander «si l'œuf est venu avant la poule ou la poule avant l'œuf ». Et de répondre qu' «il y a une corrélation sans antériorité assignable» (voir Mauss 1969: 299). Sur la position de Piaget sur ce point, voir Berthoud et Busino (1991).

6 Il ne s'agit nullement de promouvoir une opposition radicale entre nature et culture. Par exemple, Lévi-Strauss, qui passe pour celui qui a défendu avec force une telle opposition, insiste sur le fait que l'être humain est un «être vivant» (1973: 53).

7 «Aussi longtemps qu'il n'y a que deux, il n'y a pas de société. Il doit y avoir un troisième terme » (Castoriadis 1986: 54).

8 Sur la portée paradigmatique du don de Mauss comme relation triadique, voir Descombes (1996: 237). 
sociales et plus spécifiquement de la sociologie. Mais aujourd'hui, non seulement on s'interroge pour savoir si un tel concept est encore essentiel et pertinent, mais on va jusqu'à proclamer qu'il serait tout simplement dénué de sens, sauf pour un usage propre au sens commun.

Plusieurs auteurs affirment qu'ils renoncent au concept de «société», pour le remplacer, par exemple, par des notions en vogue comme flux ou réseaux (voir Urry 2000). Un sociologue comme Touraine défend une voie proprement asociologique, en affirmant que la sociologie ne peut plus être le savoir dont l'objet serait le social ou la société (voir 1987 et 1998). Pour lui, en effet, «le triomphe du capitalisme imposa l'idée d'un affaiblissement des contraintes sociales et politiques » et «la différenciation croissante entre les sous-systèmes sociaux, chacun possédant sa logique propre, devenant étrangers les uns aux autres enlève tout contenu à l'idée même de société » (1998: 123). De même, pour Touraine encore, le processus de la globalisation entraînerait des effets au niveau social et politique. Telles seraient les tendances à une déliaison du lien social et à une fragilisation de l'Etat-nation (1998: 130-31).

Dans une telle perspective, toute idée du social ou de la société se ramène à une métaphore du marché. Selon une représentation diffuse, toute relation entre personnes et entre groupes serait régulée selon la logique de l'offre et de la demande. Une manière de faire disparaître toute trace de relation sociale, ramenée à de stricts échanges calculés. Une telle dérive individualiste est diversement qualifiée de dépersonnalisation, de désymbolisation, de désocialisation. Pour d'autres, elle est vue de manière positive comme une avancée dans la voie d'une rationalisation des relations humaines. Mais pour les uns comme pour les autres, l'individu, dans ses rapports avec l'Etat et le marché, se sent à ce point libéré de toute obligation interpersonnelle qu'il peut en arriver à penser qu'il n'est plus «en société», mais dans une atmosphère marchande propre à permettre l'optimisation de toute chose, dans une ambiance de concurrence généralisée.

Dans de telles conditions, les sciences sociales, dans leur vocation à penser l'homme et la société dans le temps et dans l'espace, sont de plus en plus confrontées à un monde dont l'intelligibilité semble devenue insaisissable, ou du moins envisageable de manière parcellaire, au point de verser dans l'insignifiance. Marquée par des formes multiples de violence et un sentiment accru d'insécurité, des relations interpersonnelles aux rapports entre Etats et entre entités religieuses et culturelles, la vie humaine semble s'inscrire toujours davantage dans de purs rapports de force. Ou encore, elle se réduit à une lutte absurde et déshumanisante pour l'acquisition infinie du pouvoir et de la richesse.

Même en relativisant cette perception d'une déliaison générale, le lien social semble devoir se construire dans le seul temps présent. Disparaît ainsi toute référence au passé, vu comme dépassé et à l'avenir, en raison de l'absence de toute attente explicite. Ce possible enfermement dans un présent sans véritable mémoire et sans projet, ou sans perspective d'avenir, permettrait de comprendre pourquoi la cohésion sociale est si fragile. La double relation au passé et à l'avenir, constitutive de toute société, serait donc en crise. Comment une société, sans valeurs partagées inscrites dans l'histoire et dans les traditions culturelles, mais aussi sans véritable vision d'avenir, pourrait-elle orienter ses membres pour donner un sens à leur existence et leur permettre de vivre ensemble? Faut-il penser que chaque individu, bien que sans repère et sans perspective, soit à 
proprement parler le maître de sa vie et admettre ainsi la viabilité d'un pluralisme extrême des valeurs?

Il serait tout à fait réducteur d'en rester à une évaluation aussi rapide et simplificatrice. Contre ce désordre destructeur et contre l'absence de tout horizon mobilisateur, des forces sont à l'œuvre pour tenter d'ordonner le monde d'une certaine manière et pour y donner un sens.

Mais prises dans une telle confusion de mouvements et de contre-mouvements, les sciences sociales sont-elles encore en mesure d'élaborer des savoirs propres à éclairer de manière significative les multiples ambivalences, paradoxes et contradictions des pratiques individuelles et collectives? Ne faudrait-il pas plutôt parier sur la disparition de ces sciences? Ou tout au moins, ne faudrait-il pas constater une tendance toujours plus forte visant à réduire les sciences sociales à des disciplines pragmatiques, centrées sur la fonctionnalité du monde dans un univers de sens donné?

Sans pouvoir donner des réponses assurées sur ces questions proprement existentielles, il semble bien que la raison d'être de ces savoirs, partiellement imposée et largement acceptée, est de contribuer à apporter des solutions à des problèmes définis dans les champs politico-administratif et économique. Une telle pratique fragmentaire des sciences sociales favorise, voire impose, une «attitude technique». Et du même coup, l'exigence de la réflexion, de l'interrogation fondamentale et des spéculations théoriques tend à disparaître, ou tout au moins à être marginale. Faut-il alors penser que la seule figure reconnue soit celle du technicien du social, spécialisé dans la collecte et le traitement de données, en vue d'une hypothétique aide à la décision? Mais plus radicalement, soumises aux exigences accrues de la politique de la recherche et de la formation, les sciences sociales sont fragilisées face aux domaines privilégiés des technosciences ou de l'économie.

\section{LA DÉMARCHE COMPARATIVE: CONDITION D'UNE SCIENCE SOCIALE AUTRE?}

Et pourtant le développement d'une science sociale responsable et proprement inventive supposerait la valorisation d'une interrogation fondamentale sur l'être humain et la société, dans le sillage de toute une tradition occidentale. Depuis plusieurs siècles, une élite intellectuelle très minoritaire s'est efforcée de connaître d'autres sociétés que la sienne, pour en retour s'interroger sur ses propres fondements culturels. Nul doute que la modernité, au contraire des autres cultures et dans le sillage de la Grèce ancienne, a toujours tenté de connaître d'autres sociétés. Mais cette connaissance accrue a permis d'abord d'exercer de multiples formes de violence à travers le monde. La modernité politique et économique s'est ainsi manifestée par la conquête, la domination et l'exploitation, sans compter la violence religieuse de la conversion. Il n'en reste pas moins que cette manière d'imposer la réalisation d'un seul monde, par l'usage de la force souvent la plus brutale et légitimée par l'alibi d'une «mission civilisatrice», ou encore du «fardeau de l'homme blanc», ne 
constitue pas la seule modalité de la relation de la modernité avec l'altérité culturelle? .

Autant dire que ce désir ou cette passion de connaître, propre à la modernité, permet, entre autres, une pratique de la décentration culturelle, en relativisant la représentation dominante de la modernité, défendue comme une nouveauté historique et culturelle radicalement différente, ou comme un absolu, à partir duquel toute réalité sociale et historique serait évaluée et hiérarchisée ${ }^{10}$. Contre ce conformisme ambiant, la tâche qui incomberait à une science sociale renouvelée serait celle de la critique comme condition préalable à toute tentative constructive. Ce moment constructif supposerait de pouvoir prendre ses distances avec les prescriptions de la culture moderne. A cette condition seulement, l'horizon intellectuel serait suffisamment dégagé pour envisager de possibles éléments universellement partagés, ou encore des invariants socio-anthropologiques ${ }^{11}$. C'est dire qu'un «savoir élaboré dans les limites d'une culture définie ne peut s'arroger le droit à l'universalité [...] Un savoir légitime et pertinent sur l'homme et la société doit impérativement concevoir l'humanité à la fois dans son unité et dans sa diversité. Reconnaître dans le présent la permanence du passé et dégager les principes de notre condition humaine, au-delà de la diversité des sociétés et des cultures, c'est rejeter toute distinction radicale entre l'histoire, l'anthropologie et la sociologie. Telle devrait être l'exigence primordiale pour contribuer à construire un savoir effectif sur la complexité humaine» (Berthoud et Busino 2000: 13-14).

Une telle démarche devrait constituer la seule voie possible pour penser l' «unité-dans-la-diversité» de l'humanité. Elle suppose une mise en perspective réciproque des sociétés et des cultures et, de manière ultime, un éclairage sur la condition humaine. Merleau-Ponty a exposé très brièvement, mais très clairement, les exigences d'un tel savoir comparatif. Pour lui, «le savoir sera fondé sur

Une telle ambivalence se retrouve, par exemple, dans la controverse entre Caillois et Lévi-Strauss. Pour le premier, «la curiosité à l'égard des autres cultures m'apparaît essentielle. Si l'on me demandait [...] de désigner la supériorité principale et, si possible, la supériorité incontestable de la civilisation occidentale, je répondrais sans hésiter que c'est d'avoir enfin produit des ethnographes» (1955: 65). Dans sa réponse, Lévi-Strauss rétorque qu' «une juste appréciation des immenses conquêtes de l'Occident ne m'empêche pas de percevoir l'étrange paradoxe qui lui a fait créer les ethnographes au moment même où il entreprenait la destruction de l'objet des études qu'il leur reconnaît; ni de prendre conscience du rôle d'alibi que nous sommes contraints à jouer » (1955: 1214).

10 Interroger les vérités situées de la culture dite moderne ne signifie en aucune manière une défense du relativisme enfermant chaque culture dans les limites de ses propres normes. Mais refuser l'irréductibilité des cultures n'implique pas nécessairement de verser dans l'orthodoxie de l'universalisme abstrait. Une démarche pleinement comparative devrait porter, de manière ultime, sur l'unité fondamentale de l'humanité.

${ }^{11}$ Faut-il penser que même s'il était possible de dégager des invariants, ces derniers ne nous apporteraient rien du point de vue théorique et épistémologique, en raison de leur abstraction et donc du fait qu'ils seraient non-opératoires. Sauf à rechercher ces invariants à partir de la question majeure qui porte sur le sens de l'humanité de l'homme. Une réponse, fondée ici sur la contribution de Marcel Mauss, voit dans le don, comme terme générique, un phénomène universel, même s'il s'actualise sous des formes variables selon les contextes culturels et historiques. Avec le don, dans une perspective maussienne, l'humanité, partout et toujours, se constitue à partir d'une telle matrice, ou d'un tel invariant universel socio-anthropologique. 
ce fait irrécusable que nous ne sommes pas dans la situation comme un objet dans l'espace objectif, et qu'elle est pour nous principe de curiosité, d'investigation, d'intérêt pour les autres situations, comme variantes de la nôtre, puis pour notre propre vie, éclairée par les autres, et considérée cette fois comme variante des autres, finalement ce qui nous lie à la totalité de l'expérience humaine, non moins que ce qui nous en sépare» (1960: 137-138).

Nécessairement conditionnée par notre univers culturel, une telle comparaison vise à dégager un savoir sur les autres et simultanément à nous comprendre nousmêmes, ou tout au moins à nous voir autrement que ce que nous croyons être. Cette idée n'est certes pas nouvelle, comme peuvent l'attester les œuvres de Montaigne $^{12}$, Rousseau ${ }^{13}$ et Montesquieu ${ }^{14}$, pour ne citer que ces trois précurseurs majeurs ${ }^{15}$.

Mais le recours à quelques auteurs contemporains devrait s'imposer, pour saisir pleinement les exigences méthodologiques et théoriques d'une comparaison effective. Avec Mauss, entre autres, nous ne pouvons plus considérer les diverses formes d'altérité culturelle simplement comme un passé dépassé, mais comme un miroir, qui devrait nous faire prendre conscience de ce que nous refoulons, quand nous croyons pouvoir nous définir exclusivement comme des êtres individualistes et une société libre-échangiste, par exemple. Une vision aussi réductrice de nousmêmes constitue un obstacle majeur pour toute démarche en sciences sociales.

Pour la comparaison fondamentale, prônée et pratiquée par Dumont (1983: 17), les difficultés sont encore accrues. Inévitablement, la comparaison est d'abord une traduction, surtout quand il s'agit de mettre en rapport deux entités éloignées dans le temps et dans l'espace. Les manières de penser et d'agir des autres ne peuvent s'exprimer que dans notre propre langage. Ou encore, nos idées sur le monde ne peuvent se formuler que dans les limites de nos catégories d'analyse. Aussi, comment comprendre l'autre à partir de nos propres termes de réfé-

12 Dans le chapitre «Des cannibales» de ses Essais (1580), Montaigne affirme: «Il n'y a rien de barbare et de sauvage en cette nation, à ce qu'on m'en a rapporté, sinon que chacun appelle barbarie ce qui n'est pas de son usage; comme de vray, il semble que nous n'avons autre mire de la vérité et de la raison que l'exemple et idée des opinions et usances du païs où nous sommes » (1962: 203).

13 Dans son Essai sur l'origine des langues (1755), Rousseau énonce la règle fondamentale de toute démarche anthropologique: «Quand on veut étudier les hommes, il faut regarder près de soi; mais pour étudier l'homme, il faut apprendre à porter sa vue au loin; il faut d'abord observer les différences pour découvrir les propriétés » (1968: 89).

14 Dans De l'esprit des lois (1748), Montesquieu affirme: «J'appelle ici préjugé, non pas ce qui fait qu'on ignore de certaines choses, mais ce qui fait qu'on s'ignore soi-même» (1979: 116). De manière comparable, Malinowski n'hésite pas à avancer à la fin de son «magnum opus», Les Argonautes du Pacifique occidental (1922): «Il ne nous sera pas possible de parvenir au but suprême assigné par Socrate, qui est de se connaître soi-même, si nous ne sortons jamais du cercle étroit des coutumes, des croyances et des préjugés qui, dès notre naissance, nous emprisonne» (1963: 589).

15 Avec le redécouverte de l'antiquité gréco-romaine à la Renaissance, «on reconnaissait qu'aucune civilisation ne peut se penser elle-même, si elle ne dispose pas de quelques autres pour servir de terme de comparaison. La Renaissance a retrouvé, dans la littérature ancienne, des notions et des méthodes oubliées; mais plus encore, le moyen de mettre sa propre culture en perspective, en confrontant les conceptions contemporaines à celles d'autres temps et d'autres lieux» (LéviStrauss 1973: 319-320). 
rence, même si ces derniers sont à leur tour soumis à l'éclairage de l'altérité, appréhendée, il est vrai, à l'aide de ces mêmes termes? Faut-il voir dans ce mode de connaissance une pure tautologie? Tel est évidemment l'obstacle majeur de toute démarche comparative, qui peut aisément se réduire à un raisonnement analogique, ou même se rabattre sur une assimilation vague et confuse. Sauf, sans doute, à envisager ce va-et-vient de nous aux autres et des autres à nous à l'intérieur d'une dynamique, qui renforce progressivement la qualité comparative des termes de référence.

Plus fondamentalement, il ne s'agit pas d'une simple question de vocabulaire. Une comparaison-traduction, établie, entre autres, à partir de catégories comme «intérêt», «échange», «capital» et «marché», généralisées à l'ensemble de la vie individuelle et collective, se fermerait à la compréhension de toute altérité, ramenée à une modalité inférieure du modèle de référence. Mais, simultanément, le danger est bien de nous méprendre sur la signification profonde de nos pratiques et de nos idées, en redoublant simplement les représentations immédiates que notre société se fait d'elle-même, ou en tombant dans le piège des préjugés les plus répandus ${ }^{16}$.

Certes, ces catégories rendent possible, pour nous, la compréhension du monde, mais entraînent paradoxalement une incompréhension des autres et finalement de nous-mêmes. Ces notions du lexique (néo)libéral trouvent un sens en dehors de la sphère proprement économique, au point de structurer une vision du monde largement partagée. Des catégories propres à orienter les pratiques individuelles et collectives, qui expriment tout à la fois une vérité humaine, un univers de sens, des normes et des valeurs spécifiques. Constitutives du langage ordinaire et largement diffusées par l'ensemble des médias, ces catégories sont reprises dans les discours savants sur l'être humain et la société. Mais le moindre travail rigoureux sur la portée des mots et sur la nécessité d'en établir la généalogie se heurte inévitablement à l'usage généralisé de mots confus et du même coup souvent interchangeables. Dans un tel conformisme intellectuel, nombre de travaux en sciences sociales ne font que redoubler la vision du monde dominante. Ils en viennent à diffuser des affirmations tenues pour vraies sans véritable argumentation.

Maints travaux en sciences sociales, faute d'une telle mise en perspective, ne sont pour ainsi dire que des répliques savantes de la manière commune et réductrice de penser le monde. Ces travaux n'apportent aucune contribution effective pour comprendre la réalité humaine et sociale; ils ne font que conforter la justification idéologique imposée par de nombreux décideurs. Contre le risque d'une telle insignifiance, une science sociale inventive se devrait aujourd'hui de poursuivre dans la voie tracée par toute une pensée anthropologique ouverte sur le monde. Encore faudrait-il expliciter clairement les conditions à partir desquelles une inventivité effective serait envisageable. A commencer par admettre que toute compréhension de la condition humaine passe par un savoir propre à concevoir les relations, toujours ambivalentes et paradoxales, entre la nature qui n'est pas

\footnotetext{
En posant que l'être humain est fondamentalement un être vivant, la comparaison avec le monde animal est tout aussi essentielle que la comparaison entre les sociétés ou entre les cultures (Berthoud et Busino 1999).
} 
réductible à un construit social et la culture, entre le passé et le présent, ou encore entre «nous» et les «autres».

\section{Institut d'anthropologie et de sociologie Université de Lausanne}

\section{RÉFÉRENCES}

Berthoud, Gérald et Giovanni Busino

1995 Pratiques sociales et Théories. Les discordes des universitaires. Genève-Paris: Droz.

2000 Paroles reçues. Du bon usage des sciences sociales. Genève-Paris: Droz.

Berthoud, Gérald et Giovanni Busino, éds

1991 «L'homme et la société. Dix ans après Piaget». Revue européenne des sciences sociales 89.

1999 «Animalité et humanité. Autour d'Adolf Portmann». Revue européenne des sciences sociales 115 .

Caillois, Roger

1955 «Illusions à rebours ». Nouvelle revue française 25 : 58-70.

Castoriadis, Cornelius

1986 Domaines de l'homme. Paris: Seuil.

Cuin, Charles-Henry

2002 «Le balancier sociologique français: entre individus et structures». Revue européenne des sciences sociales 124: 253-262.

Descombes, Vincent

1996 Les institutions du sens. Paris: Minuit.

Dumont, Louis

1983 Essais sur l'individualisme. Une perspective anthropologique sur l'idéologie moderne. Paris: Seuil.

Elias, Norbert

1991 La société des individus. Paris: Fayard.

Lévi-Strauss, Claude

1955 «Diogène couché». Les Temps Modernes 110: 1187-1220.

1973 Anthropologie structurale deux. Paris: Plon.

Malinowski, Bronislaw

1963 Les Argonautes du Pacifique occidental. Paris: Gallimard.

Mauss, Marcel

1968 Euvres. 1. Les fonctions sociales du sacré. Paris: Minuit.

1969 Euvres. 3. Cohésion sociale et divisions de la sociologie. Paris: Minuit.

1996 «L'œuvre de Mauss par lui-même». Revue européenne des sciences sociales 105: 225-

236 (première publication dans la Revue française de sociologie 20, 1979, p. 209-220).

Merleau-Ponty, Maurice 1960 Signes. Paris: Gallimard.

Montaigne

1962 Euvres complètes. Paris: Gallimard.

Montesquieu

1979 De l'esprit des lois 1. Paris: Garnier-Flammarion.

Ricœur, Paul

1985 Temps et récit 3. Le temps raconté. Paris: Seuil.

Rousseau, Jean-Jacques

1968 Essai sur l'origine des langues. Bordeaux: Ducros (première publication 1755). 
Touraine, Alain

1987 «La sociologie est-elle encore l'étude de la société ?» dans Les scientifiques parlent..., A. Jacquard, éd. Paris: Hachette, p. 189-234.

1998 «Sociology without Society». Current sociology 46 (2): 119-143.

Urry, John

2000 Sociology Beyond Societies: Mobilities for the Twenty First Century. London: Routledge. 